Rapid Communications

\title{
Nanometer-scale Patterned Surfaces for Control of Cell Adhesion
}

\author{
Makiko Goto, ${ }^{* 1, * 2}$ Takehiko Tsukahara, ${ }^{* 1, * 2}$ Kae SATo, ${ }^{* 1, * 3}$ Tomohiro Konno,*3,*4 \\ Kazuhiko IshIHARA, ${ }^{* 3, * 4}$ Kiichi SATo, ${ }^{* 5}$ and Takehiko KITAMORI $* 1, * 2, * 4 \uparrow$ \\ *1 Department of Applied Chemistry, School of Engineering, The University of Tokyo, \\ 7-3-1 Hongo, Bunkyo, Tokyo 113-8656, Japan \\ *2 Core Research for Evolutional Science and Technology, Japan Science and Technology Agency, \\ 4-1-8 Honcho, Kawaguchi, Saitama 332-0012, Japan \\ *3 Center for NanoBio Integration, The University of Tokyo, 7-3-1 Hongo, Bunkyo, Tokyo 113-8656, Japan \\ *4 Department of Materials Engineering, School of Engineering, The University of Tokyo, \\ 7-3-1 Hongo, Bunkyo, Tokyo 113-8656, Japan \\ *5 Department of Applied Biological Chemistry, School of Agriculture and Life Sciences, The University of Tokyo, \\ 1-1-1 Yayoi, Bunkyo, Tokyo 113-8657, Japan
}

\begin{abstract}
A novel cell-adhesion surface, controlled by nanometer-scale topography and chemical patterning, was developed using semiconductor fabrication methods and the formation of self-assembled monolayers. The patterned surface had a sharp contrast between the adsorption and non-adsorption of proteins and cells, and the contrast could be maintained for more than 10 days. The patterning method could easily realize a single cell array and control of the cell morphology. The nanometer-scale patterned surface could control cell adhesion and proliferation. Using the patterned surface will contribute to studies about cell-surface interactions.
\end{abstract}

(Received January 18, 2007; Accepted January 29, 2007; Published March 10, 2007)

Cell adhesion plays a critical role in adherent cells because they trigger signal transduction inside the cells, while affecting cellular growth, proliferation and differentiation. The interactions between cells and surfaces have been the focus of studies in biological and medical research fields, and many biocompatible materials having cell attachable or detachable properties have been developed. The control of cell adhesion and micro patterning of cells or proteins using cell-surface interactions has been applied to cell array and co-culture systems in order to advance such fields as drug discovery and tissue engineering. ${ }^{1,2}$

Many studies on the interactions between cells and surfaces have been reported. We concentrate on surfaces patterned at the subcellular-scale $(10-1000 \mathrm{~nm})$, the size reaching that of a focal adhesion. Focal adhesion plays a pivotal role in cell adhesion and signal transduction, and subcellular-scale patterning should allow control of the morphology, proliferation, and differentiation of a cell. ${ }^{3}$ In order to investigate cell-surface interactions precisely and to control cell adhesion, it is necessary to develop surfaces with patterns much smaller than an individual cell size, i.e., nanopatterned surfaces. Several kinds of nanopatterned surfaces for cell attachment have been fabricated. ${ }^{4-7}$

Our goal is the development of novel cell culture devices with a variety of adhesion surfaces that have defined physical and chemical properties, allowing for a systematic study of cellsurface (extracellular matrix) interactions and cellular functions, depending on cell adhesion. Moreover, when the surface is fabricated in a microchannel, a novel cell culture environment can be realized by the nanometer-scale patterned-surface and

$\dagger$ To whom correspondence should be addressed.

E-mail: kitamori@icl.t.u-tokyo.ac.jp micrometer-scale liquid phase space, and individual cells in a microchannel can be easily analyzed. Using our microfluidic techniques, we have developed a microchip-based cell culture system, ${ }^{8}$ which was applied to cell imaging ${ }^{9}$ and rapid analysis of cellular-released compounds in a flow-based analysis microchip. ${ }^{10}$ We previously reported on the fabrication of a physicochemically controlled nanopatterned-surface for cell adhesion in a microchannel by using semiconductor fabrication methods and the formation of self-assembled monolayers (SAMs) of alkanethiols, and demonstrated cell culture in a microchannel by using our microfluidic techniques. ${ }^{11}$ However, this surface could not maintain the conditions for the adsorption or non-adsorption of proteins and cells for a long time. To realize many practical cellular experiments, long-term maintenance of the surface conditions is very important.

In this study, we developed a novel nanometer-scale patterned surface that had a sharp contrast between the adsorption and non-adsorption of proteins and cells. The patterned surface had topographical and chemical patterning, maintained its ability to control protein and cell adsorption for more than 10 days, and realized a variety of conditions for cell-surface adhesion. We investigated the control of cell adhesion and proliferation on patterned surfaces.

Nanometer-scale metal patterns were fabricated on quartz glass substrates by electron-beam lithography and metal sputtering methods. The fabrication scheme was described previously. ${ }^{11}$ In brief, nanopatterns were drawn by electron-beam lithography (ELS-7500, Elionix) on a quartz glass substrate with an electron-beam resist coating. After development, titanium and gold were sputtered on the substrate, and the remaining resist was removed. Metal stripes that were $150 \mathrm{~nm}$ in height and as small as $250 \mathrm{~nm}$ in width were fabricated (Fig. 1a). 
(a)

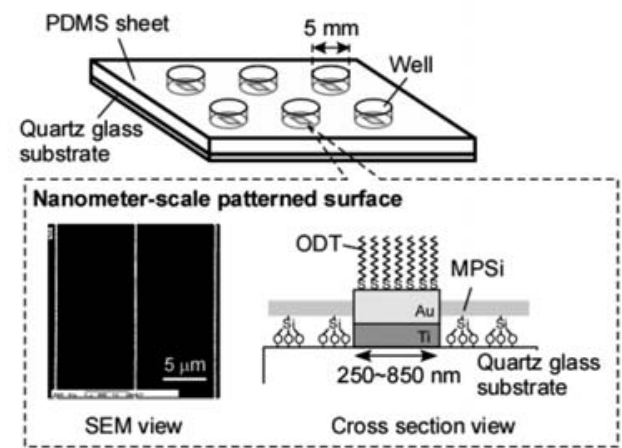

(b)

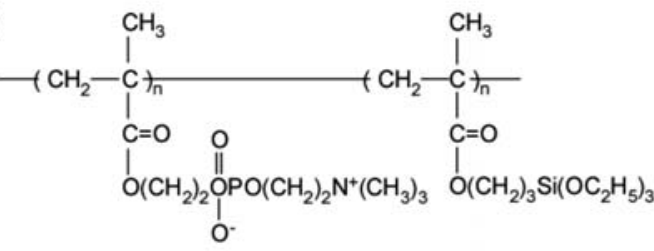

Fig. 1 (a) Diagram of the experimental tool, a scanning electron microscope (SEM) view, and a schematic cross-section view of a nanometer-scale patterned surface. White lines in the SEM view show a nanometer-scale metal pattern. (b) Chemical structure of poly[2-methacryloyloxyethyl phosphorylcholine(MPC)-co-3-methacryloyloxypropyl trimethoxysilane (MPTMSi)] (PMSi).

(a)

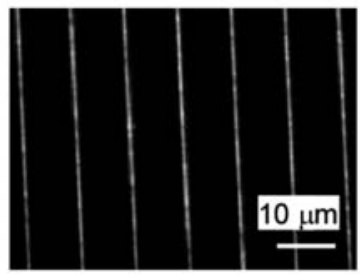

(b)

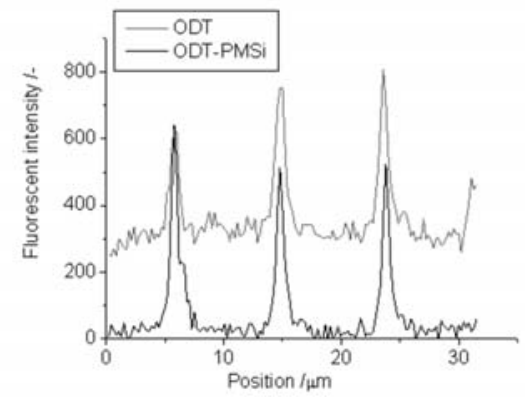

Fig. 2 (a) Fluorescent micrograph of fibronectin adsorbed on a nanometer-scale patterned surface. The surface was immunostained with an anti-fibronectin and an FITC-labeled secondary antibody. The line pattern was $550 \mathrm{~nm}$ wide and the interval was $9 \mu \mathrm{m}$. (b) Fluorescent intensities of patterned with both ODT and PMSi (black line) and ODT only modification (gray line)

Figure 1a shows a schematic diagram of chemical patterning on a topographically patterned surface. The gold patterns were coated with 1-octadecanethiol (ODT; Sigma), and then rinsed with $\mathrm{NaOH}$ and water successively. The quartz glass surface was modified with poly[2-methacryloyloxyethyl phosphorylcholine(MPC)-co-3-methacryloyloxypropyl trimethoxysilane (MPTMSi)] (PMSi) (Fig. 1b). PMSi provides an inert surface for protein adsorption and adhesion, ${ }^{12,13}$ and we used it as a barrier for protein adsorption and cell adhesion. In order to modify the surface with PMSi and fibronectin, and seed cells on
Table 1 Static contact angle of water on surfaces modified with ODT, PMSi, or both

\begin{tabular}{lcccc}
\hline Surface & $\begin{array}{c}\text { No } \\
\text { modification }\end{array}$ & $\begin{array}{c}\text { ODT } \\
\text { only }\end{array}$ & $\begin{array}{c}\text { PMSi } \\
\text { only }\end{array}$ & $\begin{array}{c}\text { ODT and then } \\
\text { PMSi }\end{array}$ \\
\hline Quartz & $10.5 \pm 3^{\circ}$ & $12.5 \pm 4^{\circ}$ & $13.7 \pm 2^{\circ}$ & $9.0 \pm 1^{\circ}$ \\
Gold & $82.6 \pm 3^{\circ}$ & $108 \pm 1^{\circ}$ & $20.5 \pm 3^{\circ}$ & $103 \pm 2^{\circ}$ \\
\hline
\end{tabular}

the surface, a polydimethylsiloxane (PDMS) sheet with some wells was made and put on the surface (Fig. 1a).

We measured the static contact angle using a DropMaster 500 (Kyowa Interface Science) on gold and quartz glass surfaces modified with ODT and PMSi. Moreover, protein adsorption on the patterned surface was investigated. The patterned surface was rinsed with phosphate-buffered saline (PBS), and then fibronectin from bovine plasma (Sigma) was adsorbed at $37^{\circ} \mathrm{C}$ for $1.5 \mathrm{~h}$. The fibronectin on the surface was visualized by immunostaining with antibodies. The surface was exposed to anti-bovine fibronectin (Biogenesis), and then to FITC-labeled secondary antibody (MP Biomedical). The surface was observed using a fluorescence microscope (IX70, Olympus) and a cooled CCD camera (CoolSnap cf. Photometrics).

Mouse fibroblast NIH/3T3 cells, or mouse preadipocyte 3T3L1 cells, were cultured on a tissue culture polystyrene dish in Dulbecco's Modified Eagle Medium containing 10\% newborn calf serum, $2 \mathrm{mM}$ L-glutamine, 100 units/ml penicillin, and 100 $\mu \mathrm{g} / \mathrm{ml}$ streptomycin (Invitrogen). The patterned surface with a PDMS sheet was put in a culture dish. After fibronectin was adsorbed on the patterned surface and washed with PBS, cells were trypsinized with a $0.25 \%$ tripsin-EDTA solution (Invitrogen), suspended in the medium, and seeded on the surface $\left(80\right.$ cells $\left./ \mathrm{mm}^{2}\right)$. After culturing for $12 \mathrm{~h}$, the PDMS sheet was peeled from the surface softly in order to supply cells with a sufficient medium and to observe them clearly in the dish. Cells on the surface were observed by using phasecontrast microscopy (TS-100, Nikon) every $12 \mathrm{~h}$.

Table 1 shows the results of contact-angle measurements on gold and quartz glass surfaces modified with ODT and PMSi. We previously noticed that when ODT modification was used on both types of surfaces, only the gold surface was modified with ODT, which became hydrophobic. ${ }^{11}$ Our present results showed that PMSi was adsorbed on both types of surfaces, but when PMSi modification was used after ODT modification, the gold surface remained hydrophobic; that is, the ODT layer could block PMSi adsorption on the gold surface.

In order to investigate protein adsorption on a nanometerscale patterned surface, the adsorbed fibronectin was immunostained and observed with a fluorescent microscope (Fig. 2a). Figure 2b shows the fluorescent intensities of linescanned points on two kinds of surfaces: one is a patterned surface (black line), and the other is a surface modified only with ODT, adsorbed fibronectin, and then immunostained (gray line). Both surfaces obtained clear differences of the intensities between a gold surface and a quartz glass surface. The intensities were obtained by subtracting the intensities of the same substrates without any modification, and the baseline meant that there was no labeled antibody on that point. On a quartz glass surface modified with PMSi, the intensity was nearly on the baseline, and there were very few fibronectin and antibody molecules; on the other hand, fibronectin or two kinds of antibody were adsorbed on a bare quartz glass surface. PMSi formed a phospholipid membrane-like surface, which was similar to a cell membrane and inert to the adsorption of 


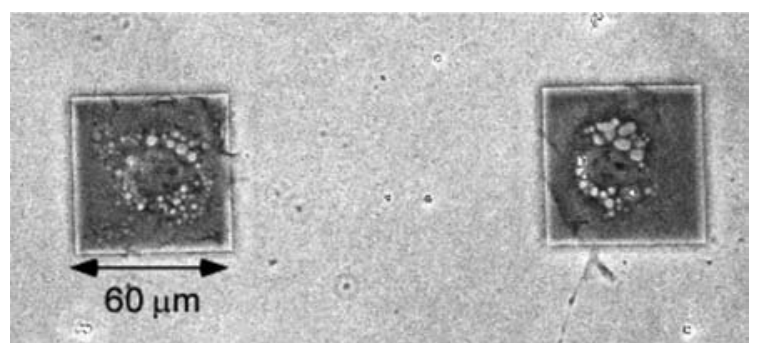

Fig. 3 Micrographs of NIH/3T3 cells on micrometer-scale square patterns.

proteins and cells. Chemical patterning with ODT and PMSi was effective enough to control protein adsorption. Both results of contact angle measurements and immunostaining showed that metal and chemical patterning of ODT and PMSi realized a nanometer-scale area for protein adsorption.

Figure 3 shows cells on a micrometer-scale square patterned surface. Individual cells attached on every micrometer-scale spot spontaneously. Our patterning method realized a singlecell array very easily.

Figure 4 shows micrographs of 3T3-L1 cells on a gold sheet $(\mathrm{a}-\mathrm{c})$ and a nanometer-scale stripe patterned surface, which was $550 \mathrm{~nm}$ in width and had an interval between two stripes of $9 \mu \mathrm{m}(\mathrm{d}, \mathrm{e})$. Cells on the patterned surface elongated along the stripes and proliferated. On every surface, cells proliferated and formed monolayers; then, some of the cell monolayers detached from the surfaces (Fig. 4e). When the pattern width was smaller than $750 \mathrm{~nm}$, many cell monolayers detached from the surface, and when the width was $250 \mathrm{~nm}$, cells could not attach to the surface. Figure $4 \mathrm{c}$ shows cultivation after 10 days. Cells adhered and proliferated on the ODT layer on the gold surface, and did not attach to the PMSi on the quartz glass surface, even after confluent growth. The inhibition of cell adhesion by PMSi was maintained for more than 10 days.

On the patterned surface, cell-surface adhesion would be strong enough for individual cells to adhere and proliferate, and they may make contact with each other. When cells formed a monolayer, the force balance of cell-cell adhesion and the cellsurface adhesion became unstable; then, cell monolayers detached from the surface and shrank, while the cells were still connected to each other. The surface could control cell adhesion and proliferation and provide an indicator of cellsurface adhesion.

In conclusion, we developed a nanometer-scale patterned surface that had a sharp contrast between the adsorption and non-adsorption of proteins and cells. The surface was fabricated by using semiconductor fabrication methods and chemical patterning. Nanometer-scale metal and chemical patterning of ODT and PMSi realized a nanometer-scale area of protein adsorption. The patterning provided cells with designed cell-surface interactions, and defined cell morphologies at a single-cell level. Cell adhesion and proliferation could be controlled on a nanometer-scale patterned surface.

The developed surface can control the strength of cell-surface adhesion, and we can investigate the properties of adherent cells under a variety of conditions for cell-surface adhesion while controlling both the surface conditions and the liquid phase conditions. When the surface is fabricated in a micrometerscale space, like a microchannel, precise control of the liquid phase is easy using microfluidic techniques. Moreover, a liquid microspace inside a microchip is suitable for experiments of single or countable adherent cells, and efficient cell analyses
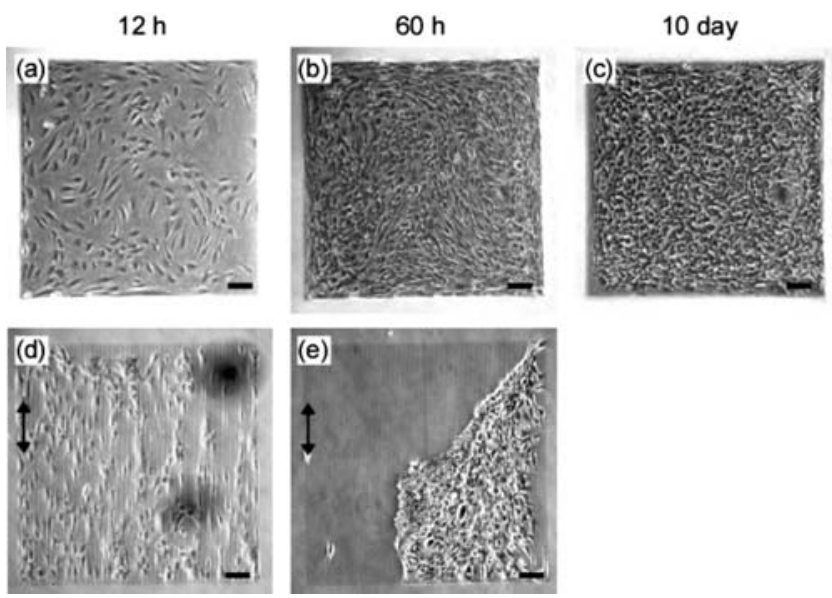

Fig. 4 Micrographs of 3T3-L1 cells on $(a-c)$ a gold sheet modified with ODT and (d, e) a nanometer-scale patterned surface. Cells were cultured for $12 \mathrm{~h}(\mathrm{a}, \mathrm{d}), 60 \mathrm{~h}(\mathrm{~b}, \mathrm{e})$, and 10 days (c). The stripe pattern was $550 \mathrm{~nm}$ in width and the interval was $9 \mu \mathrm{m}$. All scale bars show a $120 \mu \mathrm{m}$ length and arrows of $\mathrm{d}$ and e show the directions of the stripe patterns.

can be realized. A novel cell environment with nanometer-scale patterned surface and microfluidic conditions will contribute to the investigation and control of adherent cells.

\section{Acknowledgements}

A part of this research was supported by Grant-in-Aid for Scientific Research from JSPS and the Nanotechnology Project of the Ministry of Agriculture, Forestry and Fisheries of Japan.

\section{References}

1. Y. Y. Luk, M. Kato, and M. Mrksich, Langmuir, 2000, 16, 9604.

2. V. A. Liu, W. E. Jastromb, and S. N. Bhatia, J. Biomed. Mater. Res., 2002, 60, 126.

3. A. Curtis and C. Wilkinson, Trends Biotechnol., 2001, 19, 97.

4. A. I. Teixeira, P. F. Nealey, and C. J. Murphy, J. Biomed. Mater. Res., Part A, 2004, 71A, 369.

5. M. J. Dalby, M. O. Riehle, H. J. H. Johnstone, S. Affrossman, and A. S. G. Curtis, J. Biomed. Mater. Res., Part A, 2003, 67A, 1025.

6. M. J. Dalby, D. Giannaras, M. O. Riehle, N. Gadegaard, S. Affrossman, and A. S. G. Curtis, Biomaterials, 2004, 25, 77.

7. S. F. Long, S. Clarke, M. C. Davies, A. L. Lewis, G. W. Hanlon, and A. W. Lloyd, Biomaterials, 2003, 24, 4115.

8. Y. Tanaka, K. Sato, M. Yamato, T. Okano, and T. Kitamori, J. Chromatogr., A, 2006, 1111, 233.

9. E. Tamaki, K. Sato, M. Tokeshi, M. Aihara, and T. Kitamori, Anal. Chem., 2002, 74, 1560.

10. M. Goto, K. Sato, A. Murakami, M. Tokeshi, and T. Kitamori, Anal. Chem., 2005, 77, 2125.

11. M. Goto, K. Sato, M. Yamato, A. Hibara, and T. Kitamori, Proc. $\mu$ TAS 2005, 2005, 2, 1282.

12. T. Moro, Y. Takatori, K. Ishihara, T. Konno, Y. Takigawa, T. Matsushita, U. I. Chung, K. Nakamura, and H. Kawaguchi, Nat. Mater., 2004, 3, 829.

13. K. Ishihara, H. Nomura, T. Mihara, K. Kurita, Y. Iwasaki, and N. Nakabayashi, J. Biomed. Mater. Res., 1998, 39, 323. 